\title{
Bilateral extensive leg pyomyositis presenting with diabetic ketoacidosis
}

\author{
JIL SHAH, KHYATISHA SEEJORE, MICHAEL W MANSFIELD
}

Key words: pyomyositis, diabetic ketoacidosis, surgical drainage, intramuscular abscesses

\begin{abstract}
Pyomyositis is a rare and serious acute purulent bacterial infection of the skeletal muscle. Diabetes is the most important predisposing factor and, if left untreated, the infection has significant complications. We report the case of an adult male who presented acutely with a history of abdominal pain, nausea and vomiting and bilateral thigh pain. His abdominal examination was unremarkable, but a fluctuant swelling was identified in both thighs. Biochemical investigations revealed raised inflammatory markers and diagnostic chemistry of diabetic ketoacidosis. Pyomyositis was treated with intravenous antibiotics and surgical abscess drainage. MRI is the definitive investigation of choice to diagnose pyomyositis. Differential diagnoses include cellulitis, septic arthritis and deep vein thrombosis.
\end{abstract}

\section{Introduction}

Pyomyositis is a rare and serious acute purulent bacterial infection of the skeletal muscle., ${ }^{1,2}$ Early diagnosis and surgical drainage with appropriate antibiotics is required to prevent complications. ${ }^{2,3} \mathrm{MRI}$ is the definitive investigation of choice to diagnose pyomyositis. We report a case of pyomyositis which was treated with intravenous antibiotics and surgical abscess drainage.

\section{Case presentation}

A 38-year-old man with a known background of chronic pancreatitis (alcohol-related), depression and chronic hepatitis B infection presented to the emergency department after 3-4 days of abdominal pain, nausea and vomiting. He also reported bilateral thigh pain causing inability to weight-bear for 10 days prior. There was no history of trauma or fever and the patient also denied intravenous drug use. There was no prior diagnosis of diabetes. Pre-admission blood testing in primary care had shown elevated circulating D-dimer levels (1139 ng/mL: NR

Leeds Centre for Diabetes and Endocrinology, St James's University Hospital, Leeds, UK

Address for correspondence: Dr Jil Shah

Leeds Centre for Diabetes and Endocrinology, St James's University

Hospital, Leeds, UK

E-mail: jil.shah@nhs.net

Br J Diabetes 2021;21:260-262

https://doi.org/10.15277/bjd.2021.322
$<230 \mathrm{ng} / \mathrm{mL}$ ) and the referring clinician had requested exclusion of deep vein thrombosis.

On presentation he was tachycardic (heart rate $126 \mathrm{bpm}$ ) and was noted to have bilateral fluctuant thigh swelling, redness, warmth and tenderness just above the knees. Blood chemistry showed diabetic ketoacidosis (serum glucose $27.0 \mathrm{mmol} / \mathrm{L}$ (NR: 3.5-6.0), serum bicarbonate $10.9 \mathrm{mmol} / \mathrm{L}$, blood $\mathrm{pH} 7.16$ and blood ketones (hydroxybutyrate) $7 \mathrm{mmol} / \mathrm{L}$ ). He was treated in line with current UK JBDS guidelines for diabetic ketoacidosis. ${ }^{4}$

Important and relevant acute investigations were as follows: $\mathrm{Hb}$ 142 g/L (NR: 135-180), white blood cells 24.24 (NR: 4.00-11.00), neutrophils 22.1 (NR: 2.0-7.5), C-reactive protein $512 \mathrm{mg} / \mathrm{L}$ (NR: $<5)$, estimated glomerular filtration rate $>90$, serum amylase $<20$ IU/L. Blood cultures showed no growth. Echocardiogram was normal. Leg ultrasound imaging showed collections in the right biceps femoris muscle $(10 \times 7 \times 2 \mathrm{~cm})$, left sartorius muscle $(6 \times 4 \times 2 \mathrm{~cm})$ and the left external oblique muscle of the left flank $(6 \times 2 \times 2 \mathrm{~cm})$.

Magnetic resonance imaging (MRI) confirmed multiple large multiloculated intramuscular abscesses with surrounding subcutaneous tissue oedema. The collection involving the right biceps femoris $(19.5 \times 6.6 \times 4.1 \mathrm{~cm})$ was associated with marked oedema along the right sciatic nerve. Another infected collection was noted in the left vastus intermedius $(12 \times 3.8 \times 3.3 \mathrm{~cm})$ and a smaller 2.4 $\mathrm{cm}$ abscess was identified in the left biceps femoris. These are shown in Figures 1, 2 and 3. There was also mild bone marrow oedema at the posterolateral femoral condyle on the left with mild cortical irregularities and left-sided knee joint effusion and synovitis, concerning for early osteoarthritis.

Aspirate from the right thigh abscess grew methicillin-sensitive Staphylococcus aureus (MSSA) sensitive to flucloxacillin. Knee joint aspirate did not show any pus and culture was negative.

The patient had multiple bilateral large intramuscular abscesses along with concern about risk of femoral bone and knee joint involvement, coinciding with a new diagnosis of diabetes which presented as diabetic ketoacidosis. He was initially treated with intravenous flucloxacillin for 3 weeks and eventually underwent subsequent surgical drainage bilaterally. He underwent intensive physiotherapy and was discharged one week later.

\section{Discussion}

Pyomyositis is a rare bacterial infection occurring in the skeletal muscles of the body without any obvious source of infection. In the majority of cases, pyomyositis affects the lower extremities and is usually unifocal. ${ }^{5}$ Pyomyositis was first reported in the tropical regions of the world, occurring in active and healthy individuals. In temperate climates it is found in patients who are 
Figure 1. STIR (Short Tau Inversion Recovery sequence) axial image of the right thigh. This shows a large collection (arrows) involving the short head of the right biceps femoris and related to the adductor magnus with marked oedema along the right sciatic nerve (arrowhead).

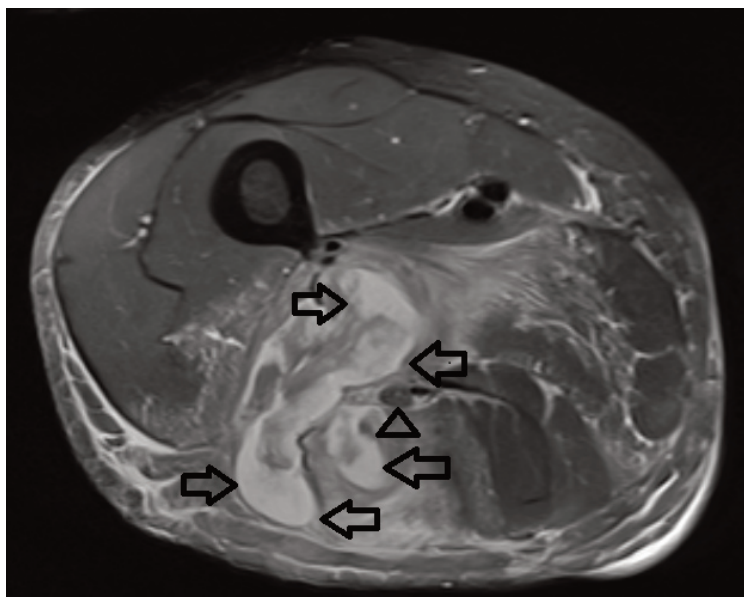

Figure 2. STIR (Short Tau Inversion Recovery sequence) large field of view coronal image of the pelvis and thighs showing multiple infected intramuscular collections with surrounding muscle, intermuscular fascial plane and subcutaneous tissue oedema. The collections are identified by arrows. The right proximal femur lesion is long standing and benign.

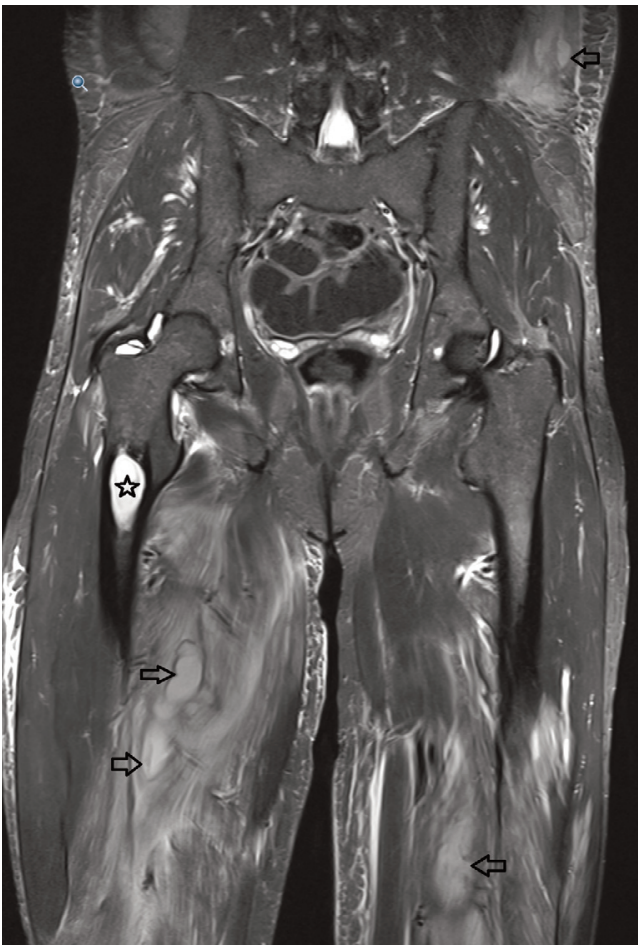

Figure 3. STIR (Short Tau Inversion Recovery sequence) coronal image of the pelvis showing the left external oblique collection. The collections are marked by arrows.

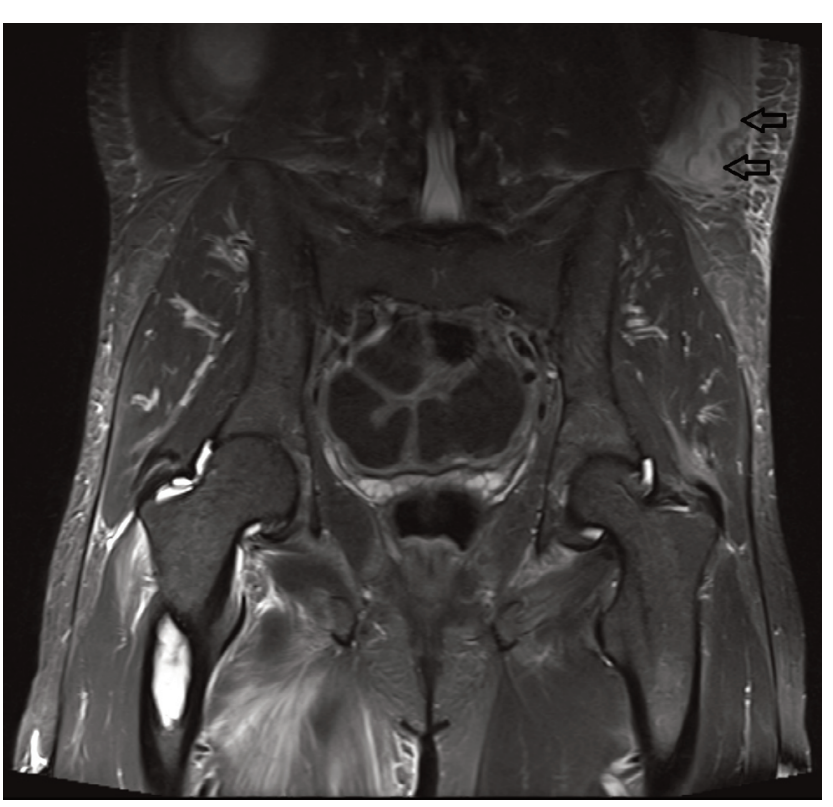

immunocompromised secondary to human immunodeficiency virus (HIV), hepatitis C or B infection, but the main risk factors typically include diabetes and trauma. ${ }^{6}$ The most common causative organisms are Staphylococcus aureus, Group B Streptococcus, Pneumococcus, Neisseria and Pseudomonas. ${ }^{2,5}$

Pyomyositis progression is divided into three stages. Stage 1 (invasive stage) is considered to be mild, comprising fever and muscle pain. At this early stage it is very difficult to identify anything significant on examination but, in a few cases, a woody texture of the affected group of muscles may be noticeable on palpation. Blood tests may reveal leucocytosis at this stage. Stage 2 (suppurative stage) usually starts 2-3 weeks after stage 1 and is characterised by fever, severe cramp-like muscle pain and localised tenderness. At this stage a fluctuant swelling may be palpable, and aspiration of the affected muscle typically shows pus. Blood tests may reveal raised inflammatory markers such as C-reactive protein, white cell count and procalcitonin. About $90 \%$ of patients seek medical attention at this stage. Stage 3 (late stage) consists of complications of pyomyositis which include systemic sepsis, disseminated infection, acute kidney injury and death if left untreated. 2,7

MRI is the gold standard diagnostic investigation. MRI helps in recognising the pathological cause, the extent of involvement of the internal structures and also helps in localising the fluid collection. If MRI is not accessible, a CT scan with or without contrast can also aid in diagnosis. Ultrasound is preferred if both CT and MRI are not available. .,8 $^{\text {On }}$

Once diagnosis is confirmed, surgical debridement and drainage together with a course of intravenous antibiotics are the recommended treatment options. For treating methicillin-sensitive 


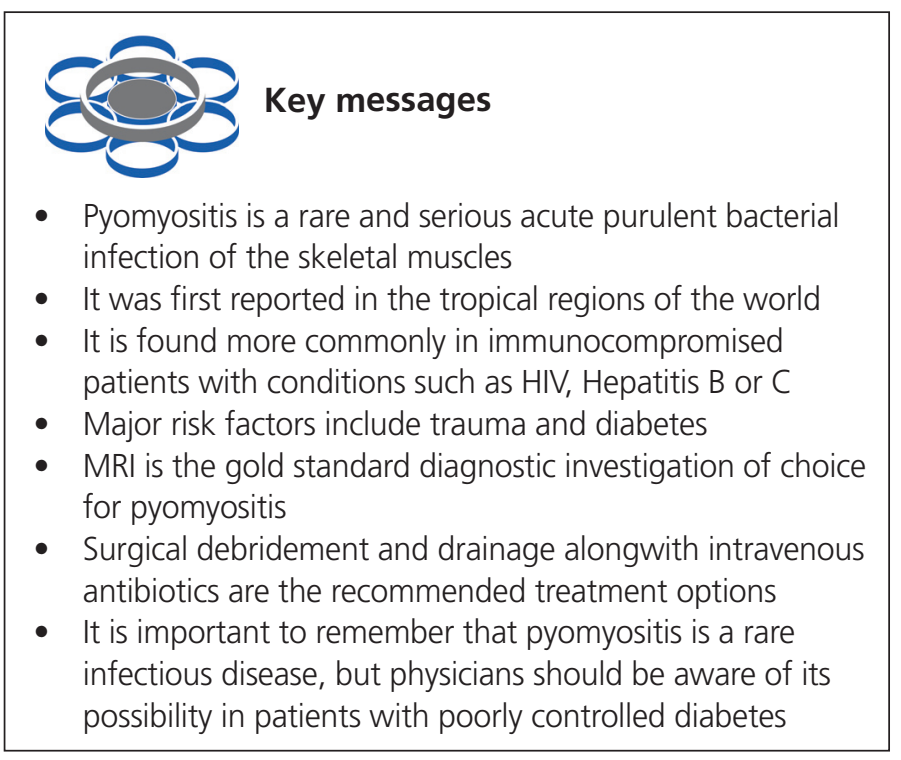

Staphylococcus aureus (MSSA) infection, flucloxacillin is the drug of choice. If pus collection identifies methicillin-resistant Staphylococcus aureus infection, vancomycin is recommended. ${ }^{9}$ It is always recommended to discuss the antibiotic of choice with the microbiology team before initiating any form of antimicrobial treatment. 7,10

Pyomyositis is a rare and serious infectious disease, and physicians should be aware of its possibility in patients with poorly controlled diabetes.
Acknowledgement Special thanks to Dr Dominic Barron, Radiology Consultant at the Leeds Teaching Hospitals NHS Trust, for helping with the radiological images.

\section{Conflict of interest None. \\ Funding None.}

\section{References}

1. Nainan A, Shagali H, Srinivasan B, Tarik A. Think pyomyositis! Pract Diabetes 2017;34:323-5. https://doi.org/10.1002/pdi.2146

2. Marath $\mathrm{H}$, Yates $\mathrm{M}$, Lee $\mathrm{M}$, Dhatariya K. Pyomyositis. J Diabetes Complications 2010;25:346-8. https://doi.org/10.1016/j.jdiacomp.2010.09.002

3. Comegna L, Guidone PI, Rossi N. Pyomyositis is not only a tropical pathology: a case series. J Med Case Reports 2016;10:372. https://doi.org/10.1186/s13256-016-1158-2

4. Joint British Diabetes Societies Inpatient Care Group. The Management of Diabetic Ketoacidosis in Adults. 2nd Edition. 2013. http://www.diabetologists-abcd.org.uk/jbds/JBDS_IP_DKA_Adults_Revised.pdf

5. Tatsuno S, Reed T, Tatsuno E, Lee C. 63-year-old man with right biceps and right pectoralis major abscesses: an unsual case of pyomyositis. BMJ 2020;13(9):e233415. https://doi.org/10.1136/bcr-2019-233415

6. Al-Dossari R, Zekri S. Failed early intervention of pyomyositis in an immunocompetent individual. Case Rep Infect Dis 2018;2018:4296976. https://doi.org/10.1155/2018/4296976

7. Chauhan S, Jain S, Varma S, Chauhan SS. Tropical pyomyositis (myositis tropicans): current perspective. Postgrad Med J 2003;80(943):267-70. https://doi.org/10.1136/pgmj.2003.009274

8. Maravelas R, Melgar TA, Vos D, Lima N, Sadarangani S. Pyomyositis in the United States 2002-2014. J Infect 2020;80(5):497-503. https://doi.org/10.1016/j.jinf.2020.02.005

9. Fox LP, Geyer AS, Grossman ME. Primary pyomyositis caused by ca-MRSA. Int J Emerg Med 2004.

10. Crum NF. Bacterial pyomyositis in the United States. Am J Med 2004; 117(6):420-8. https://doi.org/10.1016/j.amjmed.2004.03.031 